\title{
3D QSAR analysis of oxazolidinone antibacterials: can we predict?
}

\author{
Neha Gandhi* \\ School of Biomedical Sciences, Curtin University of Technology, Kent Street, \\ Bentley 6845, Western Australia \\ E-mail: nehagandhioct@rediffmail.com
}

\begin{abstract}
Three-dimensional QSAR studies for substituted aryloxazolidinones 3-9 were conducted using TSAR 3.3. The in vitro activities (MICs) of the compounds against Staphylococcus aureus and Enterococcus faecalis exhibited a good correlation with the prediction made by the model using heat of formation and LUMO energies.
\end{abstract}

Keywords: 3D QSAR, heat of formation, LUMO, antibacterial agent, aryloxazolidinone

\section{Introduction}

Multidrug resistant Gram-positive bacteria continue to pose challenges to the medicinal chemistry community. ${ }^{1}$ Linezolid $\mathbf{1}$, marketed as Zyvox ${ }^{\circledR}$, is an oxazolidinone class of antibacterial, approved for treating mostly Gram-positive bacterial infections, especially methicillin-resistant Staphylococcus aureus (MRSA), Staphylococcus Epidermidis (MRSE) and vancomycin resistant enterococci (VRE). ${ }^{2}$ Another early clinical candidate, Eperezolid 2, was discontinued from development after phase I clinical trials. While much research has been aimed at the development of novel oxazolidinones, no new members of this class have achieved regulatory approval.
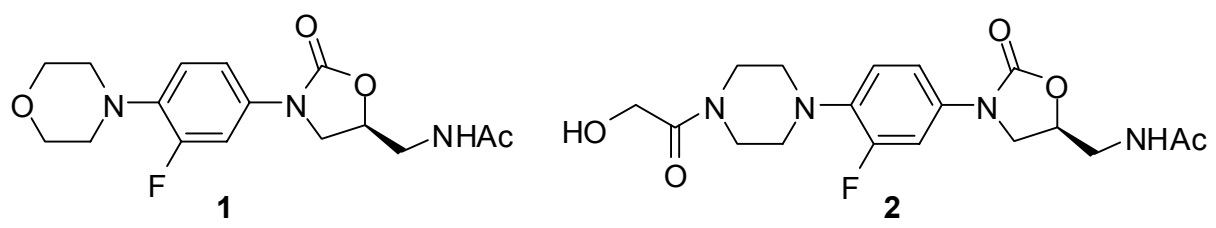

In the past, some efforts have been made to understand the structure-activity relationships of oxazolidinone antibacterial agents using comparative molecular field analysis (CoMFA), 3DQSAR and QSPR methods. ${ }^{3}$ We have already reported 3D-QSAR studies for $N-4-$ 
arylacryloylpiperazin-1-yl-phenyl-oxazolidinones $\quad 3-7 \quad$ using $\quad$ TSAR $3.3 .^{4}$ The in vitro antibacterial activities (MICs) of compounds 3-7 against Staphylococcus aureus ATCC 25923 exhibited a strong correlation with the prediction made by our model. It was observed that the activity of the compounds increases when the energy of the LUMO (Lowest Unoccupied Molecular Orbital) is lower and the heat of formation (HOF) is higher. Thus, compounds which have lower HOF become less active and, similarly, compounds having low LUMO energies are the most active and the activity decreases as the LUMO energy increases.

We decided to examine if our model (Equation 1$)^{4}$ can predict the antibacterial activity trends of different sets of compounds 8-9 for the same strain (S. aureus ATCC 25923) as well as in a different strain such as Enterococcus faecalis (E. fa) ATCC 29212.

$\log (1 / \mathrm{C})=0.006919662 * \mathrm{HOF}-0.72196823 * \mathrm{LUMO}-0.034151886 *$ Polarization YY +

$$
0.0004945533 * \text { Octupole XYZ }+4.8290181
$$

The present study aims to validate if compounds 8-9 follow the same parameters for the prediction of their activity, namely, that lower HOF and lower LUMO energies result in more potent antibacterial compounds.
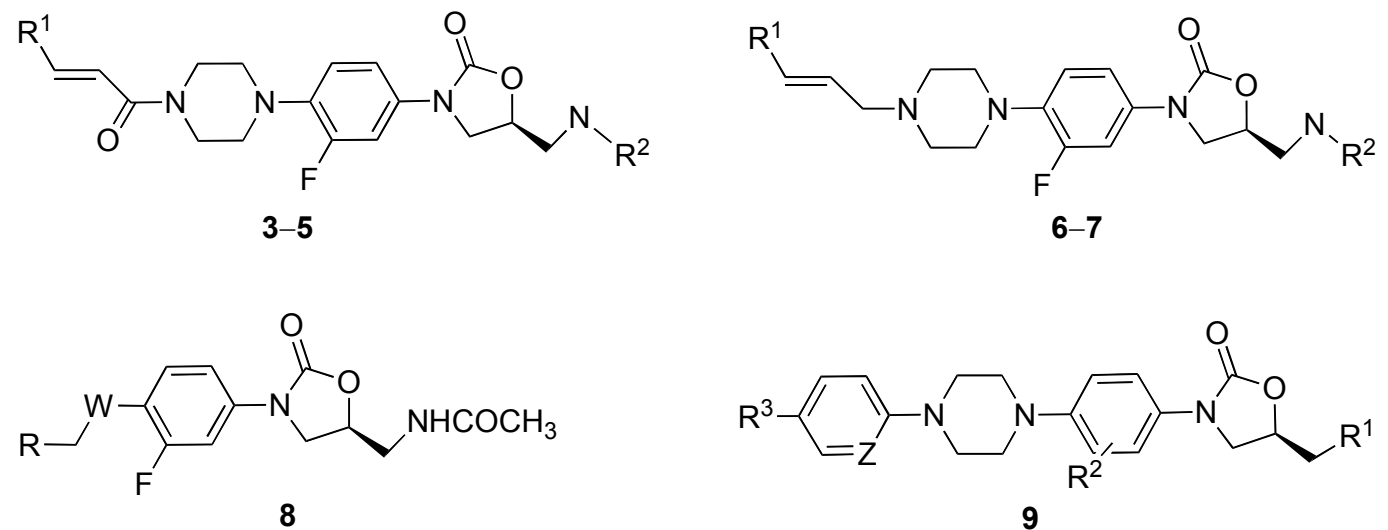

\section{Results and Discussion}

Based on Equation 1, developed using the training set of compounds reported earlier by us, ${ }^{4}$ calculations were done for a set of parameters for compounds 8a to 8aa, which is shown in Table 1. A reasonable correlation between the predicted MICs by Equation 1 and the experimental MICs reported by Das et al. ${ }^{5}$ was observed.

The predicted MICs, especially for $8 \mathbf{h}, \mathbf{8 i}, \mathbf{8 j}, \mathbf{8 m}, \mathbf{8 n}, \mathbf{8 q}, 8 \mathbf{r}$ and $\mathbf{8 u}$, were found to be 8- to 16 -fold different from the experimental values. In view of the fact that, generally, reported MICs may vary from laboratory to laboratory by a factor of one dilution (i.e. 2 to 4 or 4 to 8 ), but some of the above compounds $(\mathbf{8 h}, \mathbf{8 i}, \mathbf{8 j}, \mathbf{8 m}$ and $\mathbf{8 r})$ needed special deliberation.

Therefore, a new equation (Equation 2) was developed using randomly selected compounds from the training set shown in Table 1 and then used to predict the MIC values for the test 
compounds $8 \mathbf{b}, \mathbf{8 h}, \mathbf{8 i}, \mathbf{8 j}, \mathbf{8 m}, \mathbf{8 n}, \mathbf{8 q}, \mathbf{8 r}$ and $8 \mathbf{u}$. However, the data sets are not suited for QSAR analysis because compounds $\mathbf{8}$ have very small variance in the biological activities. Nonetheless we wanted to validate the hypothesis that the antibacterial activities follow a trend based on HOF and LUMO energies. The new 3D QSAR model that was obtained is:

$$
\log (1 / \mathrm{C})=0.0068 * \mathrm{X} 1-0.7457 * \mathrm{X} 2+2.5496
$$

where $\mathrm{X} 1$ is Heat of Formation and X2 is LUMO and the statistical parameters for above Equation 2 are: $\mathrm{s}$ value $=0.2614 ; \mathrm{F}=18.3399 ;$ regression coefficient $\mathrm{r}=0.8507 ; \mathrm{r}^{\wedge} 2=0.7237 ;$ cross validation, $\mathrm{r}^{\wedge} 2(\mathrm{CV})=0.6045$.

With the help of Equation 2, we predicted MIC values for all the compounds (shown in Table 1). Surprisingly, it was found that the predicted MIC values based on both Equations 1 and 2 were significantly different from the experimental values for the same set of compounds $(\mathbf{8 h}, \mathbf{8 i}$, $\mathbf{8 j}, \mathbf{8 m}, \mathbf{8 n}, \mathbf{8 q}, \mathbf{8 r}$ and $\mathbf{8 u}$ ). Thus, a re-examination of the SAR of these compounds was done and it was found that both Equations 1 and 2 are not capable of predicting the difference between the positional isomers. For example, when we compare $\mathbf{8 g}, \mathbf{8 q}$ and $\mathbf{8 r}$, the predicted MICs remain more or less the same $(\mathbf{8 g}, 1.33 ; \mathbf{8 q}, 1.06 ; \mathbf{8 r}, 0.6 \mu \mathrm{g} / \mathrm{mL})$, whereas observed MIC values vary significantly $(\mathbf{8 g}, 1 ; \mathbf{8 q}, 8 ; \mathbf{8 r}, 8 \mu \mathrm{g} / \mathrm{mL})$. Furthermore, the equation appears to be inadequate, especially for compounds having powerful electron withdrawing groups such as $\mathrm{CN}(\mathbf{8 h}$ : observed MIC of 16 vs. $0.8 \mu \mathrm{g} / \mathrm{mL}$ predicted with Equation 1 or $2.24 \mu \mathrm{g} / \mathrm{mL}$ predicted with Equation 2) or $\mathrm{NO}_{2}(\mathbf{8 n}$ : observed MIC of $16 \mathrm{v} / \mathrm{s} 1.48 \mu \mathrm{g} / \mathrm{mL}$ predicted with Equation 1 or 2.10 $\mu \mathrm{g} / \mathrm{mL}$ predicted with Equation 2) (see Table 1).

We also considered that it would be interesting to study if Equation 1 can predict the activity of compounds with a different structural scaffold. Therefore, a study of compounds 9a to 9ac, as reported by Jang et $a l .{ }^{6}$ (Table 2), was undertaken. A very good correlation between the predicted MIC using Equation 1 and the observed MIC (compound 9d to 9ac) was found, except for compounds 9a-9c (observed MIC $>64 \mu \mathrm{g} / \mathrm{mL}$ versus predicted MIC 2-3 $\mu \mathrm{g} / \mathrm{mL}$ ). Another equation was developed using 23 compounds listed in Table 2 as training set in order to predict the MIC of the remaining test compounds 9m, 9o, 9s, 9v, 9ab and 9ac. However, the data sets are not suited for QSAR analysis because they have significantly different $R_{1}$ residues and 15 compounds have very small variance in the biological activities. However, we wanted to investigate if compounds with a different scaffold that have antibacterial activities follow a trend based on HOF and LUMO energies.

The 3D QSAR model developed is as follows:

$$
\log (1 / C)=0.0070 * X 1-3.1117 * X 2-0.1317 * X 3+9.3684
$$

where $\mathrm{X} 1$ is Heat of Formation, $\mathrm{X} 2$ is LUMO and X3 is Polarization YY and the statistical parameters for above Equation 3 are: $\mathrm{s}$ value $=0.4182 ; \mathrm{F}=34.5641$; regression coefficient $\mathrm{r}=$ $0.9193 ; \mathrm{r}^{\wedge} 2=0.8451 ;$ cross validation, $\mathrm{r}^{\wedge} 2(\mathrm{CV})=0.7246$. 
Table 1. Prediction of antibacterial activity of piperazinylphenyloxazolidinone ${ }^{5}$

\begin{tabular}{|c|c|c|c|c|c|c|c|c|c|c|c|c|}
\hline \multirow[b]{2}{*}{ 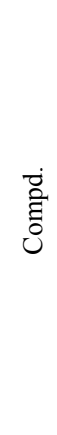 } & \multirow[b]{2}{*}{ W } & \multirow[b]{2}{*}{$\mathrm{R}$} & \multirow[b]{2}{*}{ 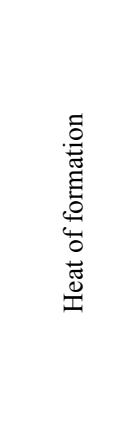 } & \multirow[b]{2}{*}{ LUMO } & \multirow[b]{2}{*}{ 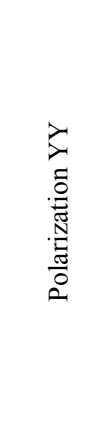 } & \multirow[b]{2}{*}{$\begin{array}{c}\text { Octupole } \\
\text { XXZ }\end{array}$} & \multirow[b]{2}{*}{$\begin{array}{c}\text { Octupole } \\
\text { ZZZ }\end{array}$} & \multicolumn{3}{|c|}{ S. aureus ATCC 25923} & \multicolumn{2}{|c|}{$\begin{array}{c}\text { E. faecalis } \\
\text { ATCC } 29212\end{array}$} \\
\hline & & & & & & & & 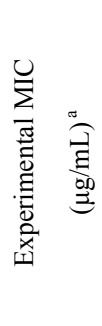 & 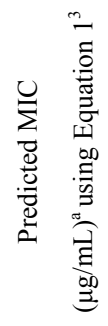 & 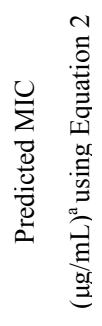 & 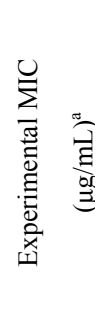 & 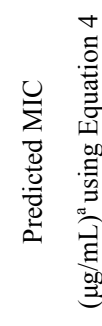 \\
\hline $8 \mathbf{a}$ & piperazine & 5 -furan & -138.857 & -0.483 & 57.547 & 51.640 & -23.679 & 8 & 2.33 & 4.52 & 8 & 11.481 \\
\hline $8 b$ & piperazine & $\begin{array}{l}\text { 5-furan-2- } \\
\text { carbaldehyde }\end{array}$ & -171.408 & -0.523 & 59.461 & -41.400 & -16.974 & 1 & 3.37 & 7.45 & 8 & 22.545 \\
\hline $8 c$ & piperazine & $\begin{array}{c}\text { 5-furan-2- } \\
\text { carboxylic acid }\end{array}$ & -226.546 & -0.548 & 60.518 & 143.518 & -36.220 & $>16$ & 8.44 & 17.65 & $>16$ & 14.266 \\
\hline $8 d$ & piperazine & $\begin{array}{c}\text { ethyl 5-furan-2- } \\
\text { carboxylate }\end{array}$ & -223.475 & -0.536 & 66.271 & 162.936 & -50.090 & $>16$ & 15.49 & 21.07 & $>16$ & 14.492 \\
\hline $8 e$ & piperazine & $\begin{array}{l}\text { 5-furan-2- } \\
\text { carbaldehyde } \\
\text { oxime }\end{array}$ & -129.767 & -0.500 & 60.600 & 53.342 & -17.558 & 2 & 3.25 & 4.21 & 8 & 10.688 \\
\hline $8 f$ & piperazine & $\begin{array}{c}\text { (5-furan-2- } \\
\text { ylmethylene)- } \\
\text { hydrazine }\end{array}$ & -101.315 & -0.541 & 62.199 & -156.048 & 30.532 & 2 & 1.69 & 2.50 & 8 & 18.187 \\
\hline $8 g$ & piperazine & 2-nitro-5-furan & -145.305 & -1.030 & 58.786 & 7.109 & -3.658 & 1 & 1.33 & 1.96 & 2 & 2.306 \\
\hline $8 \mathbf{h}$ & piperazine & $\begin{array}{l}\text { 5-furan-2- } \\
\text { carbonitrile }\end{array}$ & -100.149 & -0.566 & 61.492 & 45.497 & -11.147 & 16 & 0.80 & 2.24 & 16 & 6.386 \\
\hline $8 \mathbf{i}$ & piperazine & 2-bromo-5-furan & -125.693 & -0.499 & 58.659 & 70.605 & -24.257 & $>16$ & 1.29 & 4.21 & $>16$ & 10.425 \\
\hline $8 \mathbf{j}$ & piperazine & 2-chloro-5-furan & -142.786 & -0.498 & 58.827 & 60.863 & -34.639 & $>16$ & 2.56 & 5.06 & $>16$ & 12.064 \\
\hline $8 \mathbf{k}$ & piperazine & $\begin{array}{l}\text { 5-furan-2- } \\
\text { ylmethanol }\end{array}$ & -182.986 & -0.491 & 61.661 & 53.422 & 7.415 & $>16$ & 5.90 & 9.77 & $>16$ & 15.940 \\
\hline 81 & piperazine & $\begin{array}{c}\text { 5-furan-2- } \\
\text { ylmethyl acetate }\end{array}$ & -225.982 & -0.500 & 65.699 & 85.765 & -19.444 & $>16$ & 17.08 & 20.20 & $>16$ & 22.807 \\
\hline
\end{tabular}




\begin{tabular}{|c|c|c|c|c|c|c|c|c|c|c|c|c|}
\hline $8 m$ & piperazine & 2-methyl-5-furan & -147.575 & -0.474 & 60.600 & 50.328 & -24.345 & $>16$ & 3.55 & 5.42 & $>16$ & 13.368 \\
\hline $8 n$ & piperazine & $\begin{array}{l}\text { 2-nitro-5- } \\
\text { thiophene }\end{array}$ & -107.413 & -1.561 & 61.037 & 202.343 & -153.299 & 8 & 0.45 & 0.48 & 8 & 0.152 \\
\hline 80 & piperazine & $\begin{array}{l}\text { 2-nitro-1H- } \\
\text { pyrrole }\end{array}$ & -122.936 & -0.705 & 58.010 & 195.402 & -377.869 & 4 & 1.11 & 3.05 & 4 & 7.286 \\
\hline $8 p$ & piperazine & $\begin{array}{c}\text { 1-methyl-2-nitro- } \\
1 H \text {-pyrrole }\end{array}$ & -122.267 & -0.664 & 60.509 & 240.185 & -376.542 & 4 & 1.49 & 3.13 & 4 & 6.822 \\
\hline $8 q$ & piperazine & 2-nitro-4-furan & -146.711 & -1.068 & 56.872 & 228.770 & -308.339 & 8 & 1.06 & 2.04 & 8 & 1.691 \\
\hline $8 r$ & piperazine & 2-nitro-3-furan & -145.417 & -1.007 & 58.153 & -55.671 & 66.159 & 8 & 0.60 & 2.24 & 4 & 2.788 \\
\hline $8 s$ & 1,4-diazepane & 2-nitro-5-furan & -146.145 & -1.133 & 60.568 & -1.087 & -15.551 & 2 & 2.17 & 2.21 & 2 & 1.804 \\
\hline $8 t$ & $\begin{array}{l}\text { 2-methyl- } \\
\text { piperazine }\end{array}$ & 2-nitro-5-furan & -150.401 & -1.117 & 61.356 & -23.419 & -403.063 & 4 & 1.62 & 2.39 & 4 & 7.543 \\
\hline $8 u$ & $\begin{array}{c}(2 S, 6 R)-2,6- \\
\text { dimethyl- } \\
\text { piperazine }\end{array}$ & 2-nitro-5-furan & -156.006 & -1.152 & 61.695 & -272.272 & 374.691 & 16 & 1.48 & 2.10 & 8 & 2.260 \\
\hline $8 v$ & $\begin{array}{c}N \text {-methylpiperi- } \\
\text { din-4-amine }\end{array}$ & 2-nitro-5-furan & -153.601 & -1.036 & 67.825 & -37.910 & 321.593 & 1 & 2.91 & 2.59 & 0.5 & 1.169 \\
\hline $8 w$ & $\begin{array}{c}N \text {-(piperidin-4- } \\
\text { yl)acetamide }\end{array}$ & 2-nitro-5-furan & -192.507 & -1.412 & 68.340 & 300.223 & -533.654 & $>16$ & 5.97 & 5.54 & $>16$ & 1.192 \\
\hline $8 x$ & piperidin-4-amine & 2-nitro-5-furan & -154.942 & -1.151 & 64.747 & 239.549 & -70.990 & 1 & 2.66 & 2.03 & 0.5 & 0.626 \\
\hline $8 y$ & $\begin{array}{l}\text { N,3-dimethyl- } \\
\text { piperidin-4-amine }\end{array}$ & 2-nitro-5-furan & -156.755 & -1.073 & 68.380 & 93.588 & 257.501 & $>16$ & 3.28 & 2.91 & $>16$ & 0.671 \\
\hline $8 z$ & $\begin{array}{l}(1 R, 5 S, 6 S)-N- \\
\text { methyl-3-aza- } \\
\text { bicyclo[3.1.0]- } \\
\text { hexan-6-amine }\end{array}$ & 2-nitro-5-furan & -121.769 & -1.109 & 64.627 & -99.094 & -59.585 & 2 & 0.60 & 1.26 & 2 & 3.090 \\
\hline 8aa & $\begin{array}{c}\text { 1-((1R,5S,6S)-3- } \\
\text { azabicyclo- } \\
\text { [3.1.0]hexan-6- } \\
\text { yl)- } N \text {-methyl- } \\
\text { methanamine }\end{array}$ & 2-nitro-5-furan & -123.913 & -1.020 & 56.622 & -307.942 & 545.798 & 2 & 0.94 & 1.36 & 2 & 1.943 \\
\hline
\end{tabular}

${ }^{a} \mathrm{MIC}=$ Minimum Inhibitory Concentration for inhibition of the organism shown in $\mu \mathrm{g} / \mathrm{mL}$. 
Table 2. Prediction of antibacterial activity of arylpiperazinyloxazolidinones with diversification of the $N$-substituents ${ }^{6}$

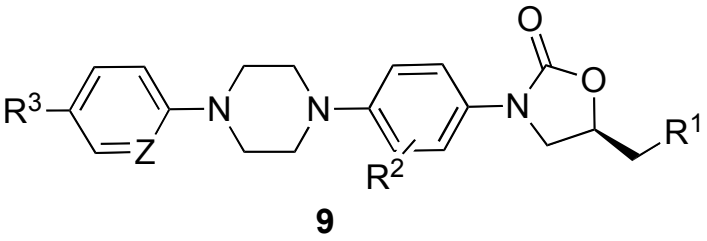

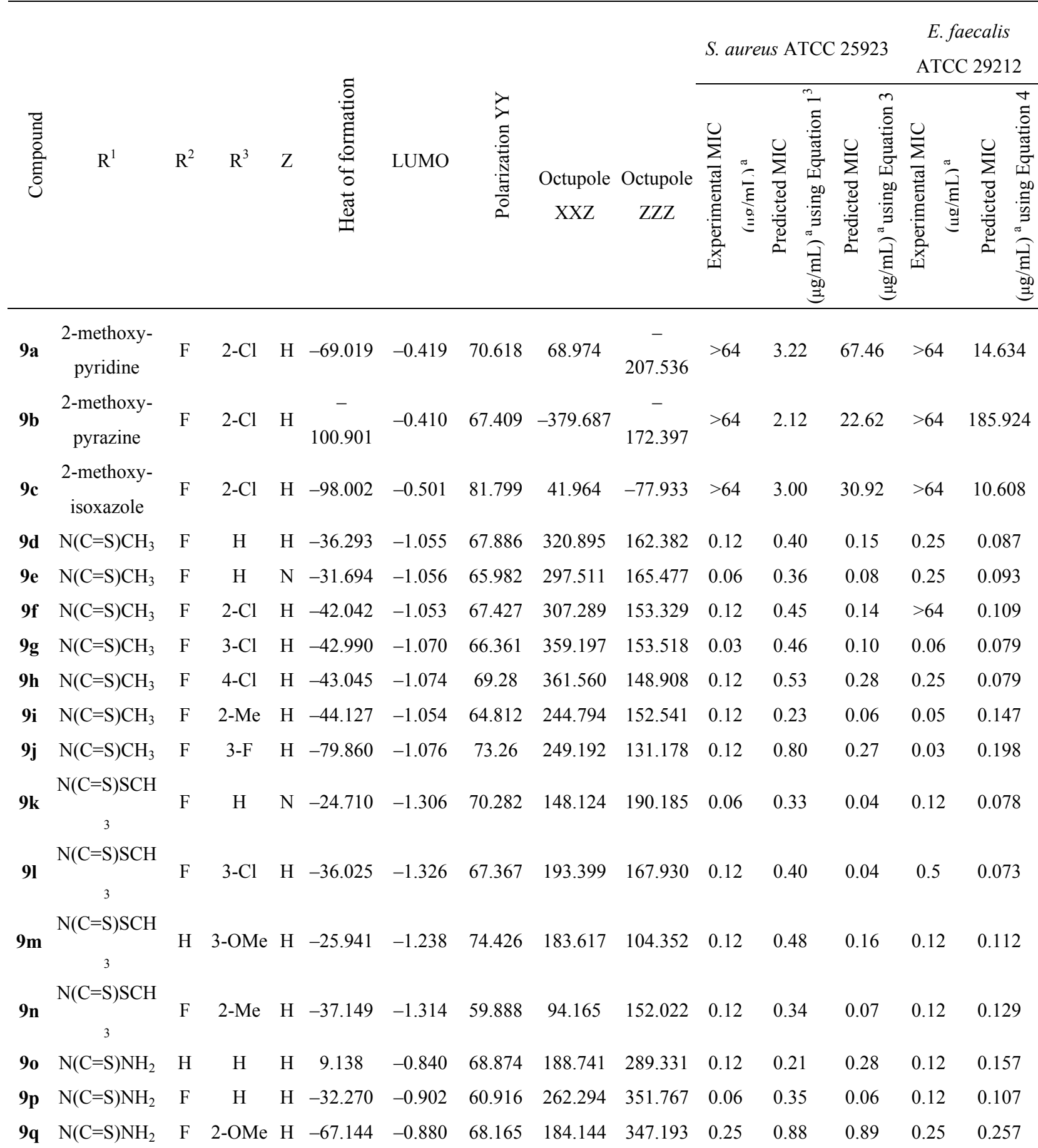




\begin{tabular}{|c|c|c|c|c|c|c|c|c|c|c|c|c|c|c|}
\hline $9 \mathbf{r}$ & $\mathrm{N}(\mathrm{C}=\mathrm{S}) \mathrm{NH}_{2}$ & $\mathrm{~F}$ & $\mathrm{H}$ & $\mathrm{N}$ & -27.651 & -0.899 & 59.444 & 253.605 & 361.004 & 0.12 & 0.32 & 0.05 & 0.25 & 0.106 \\
\hline $9 \mathrm{~s}$ & $\mathrm{~N}(\mathrm{C}=\mathrm{S}) \mathrm{NH}_{2}$ & $\mathrm{H}$ & $2-\mathrm{Cl}$ & $\mathrm{H}$ & 3.343 & -0.833 & 69.131 & 165.825 & 270.297 & 0.03 & 0.26 & 0.39 & 0.06 & 0.220 \\
\hline $9 t$ & $\mathrm{~N}(\mathrm{C}=\mathrm{S}) \mathrm{NH}_{2}$ & $\mathrm{~F}$ & $2-\mathrm{Cl}$ & $\mathrm{H}$ & -38.004 & -0.891 & 60.58 & 267.692 & 340.235 & 0.12 & 0.40 & 0.05 & 0.25 & 0.128 \\
\hline $9 \mathrm{u}$ & $\mathrm{N}(\mathrm{C}=\mathrm{S}) \mathrm{NH}_{2}$ & $\mathrm{~F}$ & $3-\mathrm{Cl}$ & $\mathrm{H}$ & -38.968 & -0.915 & 60.009 & 306.873 & 368.777 & 0.02 & 0.39 & 0.05 & 0.06 & 0.088 \\
\hline $9 v$ & $\mathrm{~N}(\mathrm{C}=\mathrm{S}) \mathrm{NH}_{2}$ & $\mathrm{~F}$ & 3-OMe & $\mathrm{H}$ & -70.177 & -0.893 & 65.554 & 292.509 & 363.029 & 0.03 & 0.93 & 0.40 & 0.06 & 0.137 \\
\hline $9 w$ & $\mathrm{~N}(\mathrm{C}=\mathrm{S}) \mathrm{NH}_{2}$ & $\mathrm{~F}$ & $4-\mathrm{Cl}$ & $\mathrm{H}$ & -39.003 & -0.907 & 62.505 & 252.591 & 360.425 & 0.12 & 0.48 & 0.10 & 0.5 & 0.123 \\
\hline $9 x$ & $\mathrm{~N}(\mathrm{C}=\mathrm{S}) \mathrm{NH}_{2}$ & $\mathrm{~F}$ & 2-Me & $\mathrm{H}$ & -40.094 & -0.902 & 64.64 & 201.658 & 338.908 & 0.12 & 0.52 & 0.15 & 0.25 & 0.169 \\
\hline $9 y$ & $\mathrm{~N}(\mathrm{C}=\mathrm{S}) \mathrm{NH}_{2}$ & $\mathrm{~F}$ & $3-\mathrm{CF}_{3}$ & $\mathrm{H}$ & $\begin{array}{c}- \\
190.460\end{array}$ & -0.932 & 53.818 & 223.300 & 338.088 & 0.25 & 2.59 & 0.16 & 0.25 & 0.584 \\
\hline $9 z$ & $\begin{array}{c}\mathrm{N}(\mathrm{C}=\mathrm{S}) \mathrm{OCH} \\
3\end{array}$ & $\mathrm{~F}$ & $\mathrm{H}$ & $\mathrm{H}$ & -70.673 & -0.822 & 61.292 & 265.441 & 133.243 & 0.25 & 0.65 & 0.21 & 0.25 & 0.406 \\
\hline $9 \mathbf{a a}$ & $\begin{array}{c}\mathrm{N}(\mathrm{C}=\mathrm{S}) \mathrm{OCH} \\
3\end{array}$ & $\mathrm{~F}$ & $\mathrm{H}$ & $\mathrm{N}$ & -66.071 & -0.813 & 67.096 & 238.542 & 167.998 & 0.12 & 1.10 & 0.97 & 0.12 & 0.414 \\
\hline $9 \mathrm{ab}$ & $\begin{array}{c}\mathrm{N}(\mathrm{C}=\mathrm{S}) \mathrm{OCH} \\
3\end{array}$ & $\mathrm{~F}$ & $3-\mathrm{Cl}$ & $\mathrm{H}$ & -77.372 & -0.835 & 65.448 & 293.138 & 131.627 & 0.12 & 1.30 & 0.89 & 0.12 & 0.385 \\
\hline 9 ac & $\begin{array}{c}\mathrm{N}(\mathrm{C}=\mathrm{S}) \mathrm{OCH} \\
3\end{array}$ & $\mathrm{~F}$ & 3-OMe & $\mathrm{H}$ & $\begin{array}{c}- \\
108.607\end{array}$ & -0.813 & 49.076 & 289.903 & 133.527 & 0.12 & 2.04 & 3.46 & 0.12 & 0.552 \\
\hline
\end{tabular}

${ }^{\mathrm{a}} \mathrm{MIC}=$ Minimum Inhibitory Concentration for inhibition of the organism shown in $\mu \mathrm{g} / \mathrm{mL}$.

The constant term in Equation 3 is dominant. Therefore, it is instructive to look at the molecular descriptors, based on $t$ value, in the regression model. Descriptors with large $|t|$ values are important in the predictive model and, as such, can be examined in order to gain some understanding of the nature of the property or activity of interest. The descriptors which had higher $t$-values and which appeared with higher frequency in previous models were selected to derive the final regression model. Table 3 indicates the statistical significance of descriptors HOF, LUMO and Polarization YY used in the derivation of Equation 3.

Table 3. Descriptors included in the model

\begin{tabular}{lcccc}
\hline \multicolumn{1}{c}{ Descriptor } & Jacknife SE $^{\mathrm{a}}$ & Covariance SE $^{\mathrm{b}}$ & t-value $^{\mathrm{c}}$ & t-probability $^{\mathrm{d}}$ \\
\hline Heat of formation (X1) & 0.0076 & 0.0032 & 2.4278 & 0.0247 \\
LUMO (X2) & 0.8491 & 0.5157 & -4.583 & 0.0001 \\
Polarization YY (X3) & 0.0245 & 0.0204 & -2.9639 & 0.0076 \\
Constant (C) & 1.5901 & & & \\
\hline
\end{tabular}

${ }^{a}$ An estimate of the standard error on each regression coefficient derived from a jack-knife procedure on the final regression model.

${ }^{b}$ Gives an estimate of the standard error on each regression coefficient derived from the covariance matrix.

${ }^{\mathrm{c}}$ Measures the significance of each variable included in the final model.

${ }^{\mathrm{d}}$ Statistical significance for t-values. 
The predicted MICs obtained using Equation 3 are shown in Table 2. We found an excellent correlation between the predicted and observed MICs. In all three equations we see a good correlation of activity with HOF and LUMO energies. Therefore, we made plots of C*HOF versus $C^{*}$ LUMO based on Equation 2, where $\mathrm{C}$ is a constant coefficient for compounds 8a-8aa (Figure 1), and for compounds 8a-8ac based on Equation 3 (Figure 2). It can be observed in these figures that all the compounds that have high HOF and low LUMO energies always have superior antibacterial activities.

Encouraged by these results, a similar equation (Equation 4 ) for Enterococcus faecalis ( $E$. fa) ATCC 29212 was developed using randomly selected analogues of compounds 8 and 9 in the training set. Compounds 8c, 8f, 8h, 81, 8z, 9e, 9h, 9k, 9r and 9x made up the test set. Compounds $\mathbf{8 n}, \mathbf{8 w}, \mathbf{8 y}, \mathbf{9 f}$ were treated as outliers. Equation 4 is:

$$
\begin{gathered}
\log (1 / C)=0.0038735112 * X 1-1.5339189 * X 2+0.0022498923 * X 3+0.001369058 * \\
X 4+1.272866,
\end{gathered}
$$

where X1 is Heat of Formation; X2 is LUMO; X3 is Octupole XXZ and X4 is Octupole ZZZ Component and the statistical parameters for above Equation 4 are: $n=37$; s value $=0.36$; $F=$ 65.16 ; regression coefficient $r=0.93 ; r^{\wedge} 2=0.88$; cross validation, $r^{\wedge} 2(C V)=0.83$.

Activity Increases

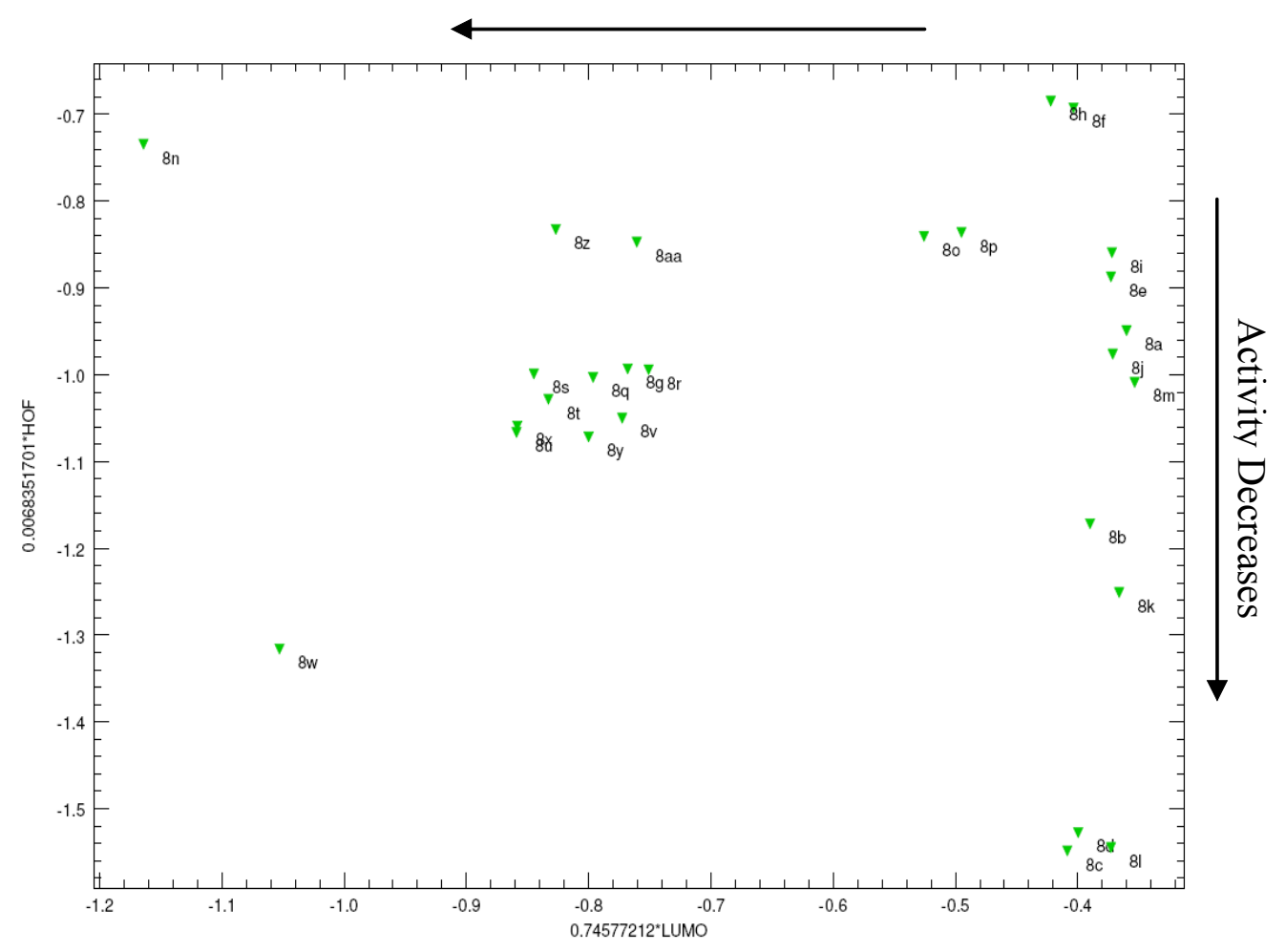

Figure 1. Plot of $\mathrm{C}^{*} \mathrm{HOF}$ vs. $\mathrm{C}^{*} \mathrm{LUMO}$ of various training and test set of compounds 8 . 
Once again, a very good correlation between the predicted $\log (1 / \mathrm{C})$ using Equation 4 and the observed $\log (1 / \mathrm{C})$ was observed for compounds 8 as well as 9 against $E$. $f a$ ATCC 29212. The results are summarised in Tables 1 and 2 . The correlation between the observed and the predicted antibacterial activities for the training set and the test set for compounds $\mathbf{8}$ and $\mathbf{9}$ using Equation 4 is shown in Figure 3.

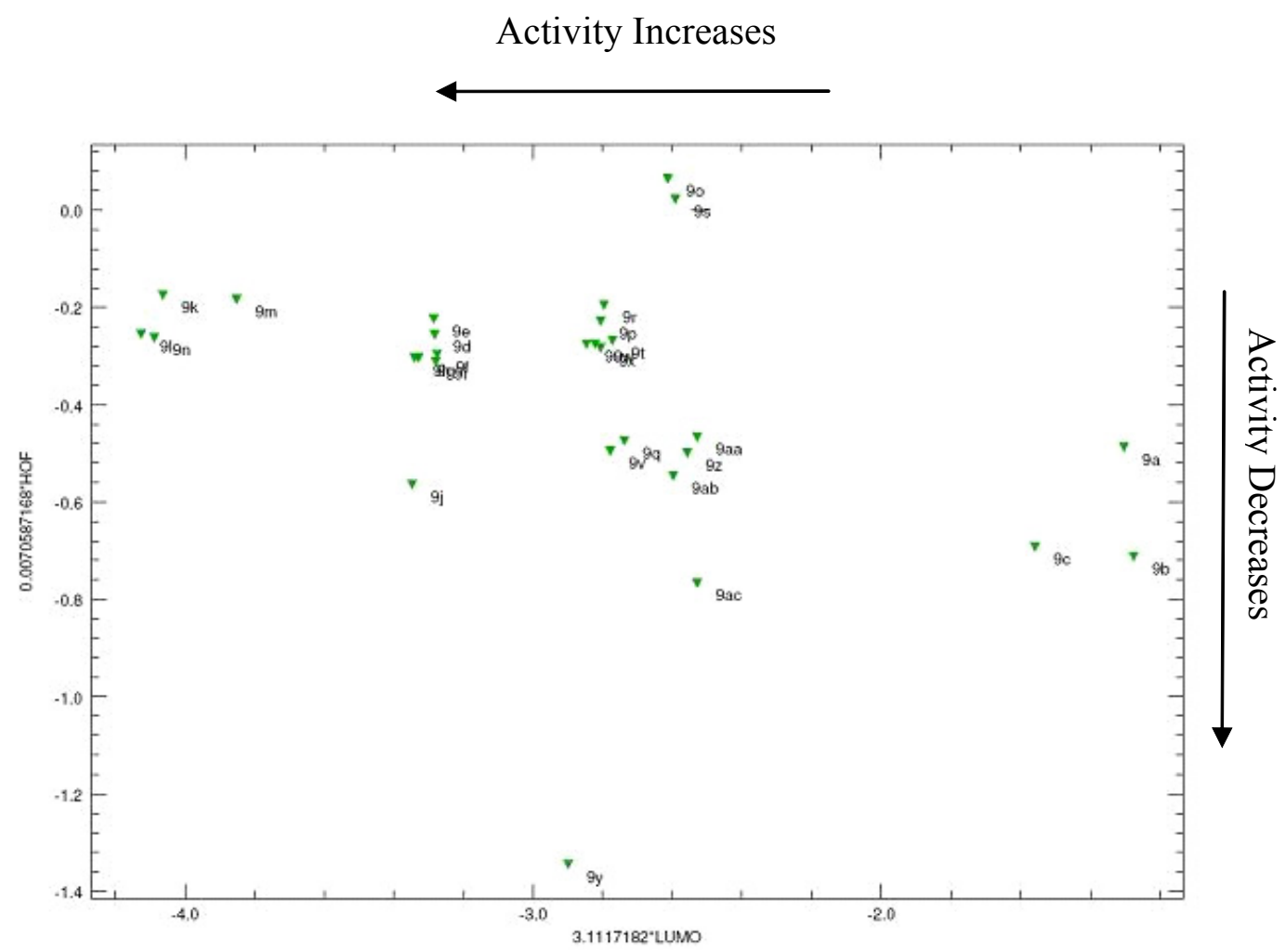

Figure 2. Plot of $\mathrm{C}^{*} \mathrm{HOF}$ vs. $\mathrm{C}^{*} \mathrm{LUMO}$ of various training and test set of compounds 9.

It was expected that such a good correlation between observed and predicted antibacterial activities will also exhibit a close correlation with HOF and LUMO energies. An analysis of the plots of $\mathrm{C}^{*} \mathrm{HOF}$ vs $\mathrm{C}^{*} \mathrm{LUMO}$ (figure and values calculated for $\mathrm{C}^{*} \mathrm{HOF}$ and $\mathrm{C}^{*} \mathrm{LUMO}$ from Equation 4 are not shown in Tables 1 and 2), where $\mathrm{C}$ is a constant coefficient, for compound 8 and compound 9 for $E$. fa ATCC 29212, clearly suggests that for both sets of compounds as the $\mathrm{C}^{*} \mathrm{HOF}$ increases and $\mathrm{C}^{*} \mathrm{LUMO}$ decreases, the antibacterial potency of compounds improves and lower MIC values are observed.

It would be worthwhile to examine the antibacterial activities of compounds 3-7 in $E$. $f a$ ATCC 29212 and verify if the values predicted (see Table 4) with Equation 4 are comparable to the observed MICs. However, to date there is no antibacterial activity data reported for compounds 3-7 for E. fa ATCC 29212. 
Table 4. Prediction of antibacterial activity of N-4-arylacryloylpiperazin-1-yl-phenyloxazolidinones $^{4}$

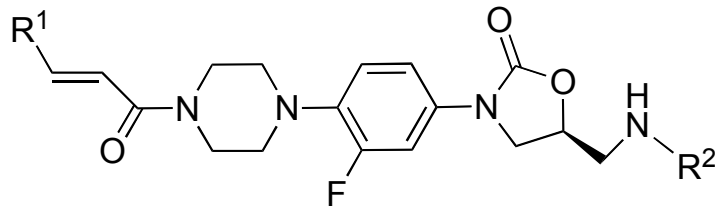

3-5

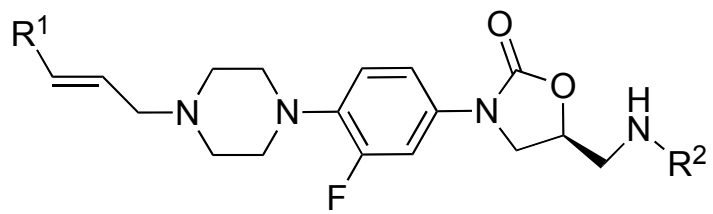

6-7

\begin{tabular}{|c|c|c|c|c|c|c|c|}
\hline Compound & $\mathrm{R}^{1}$ & $\mathrm{R}^{2}$ & 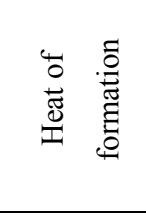 & LUMO & Oct. XXZ & Oct. ZZZ & $\begin{array}{c}\text { Predicted } \\
\log (1 / \mathrm{C})^{\mathrm{a}} \text { for } E . \\
f a \text { ATCC } 29212 \\
\text { using Equation } 4 .\end{array}$ \\
\hline $3 \mathbf{a}$ & $\mathrm{Ph}$ & $\mathrm{COMe}$ & -133.431 & -0.836 & -79.777 & 101.405 & 1.998 \\
\hline $\mathbf{3 b}$ & 4-PhOH & $\mathrm{COMe}$ & -178.186 & -0.793 & 59.534 & 5.055 & 1.940 \\
\hline $3 c$ & $3-\mathrm{PhOH}$ & $\mathrm{COMe}$ & -178.257 & -0.876 & -101.717 & 294.099 & 2.100 \\
\hline 3d & 2-thiophene & $\mathrm{COMe}$ & -122.886 & -1.213 & -53.763 & 211.167 & 2.825 \\
\hline $3 e$ & 3-thiophene & $\mathrm{COMe}$ & -125.282 & -1.000 & -51.425 & 30.985 & 2.248 \\
\hline $3 f$ & 2-furan & $\mathrm{COMe}$ & -159.744 & -0.858 & -0.136 & 260.705 & 2.326 \\
\hline $3 g$ & 3 -furan & $\mathrm{COMe}$ & -161.318 & -0.649 & 32.383 & -15.588 & 1.696 \\
\hline $3 \mathbf{h}$ & 2-1H-pyrrole & $\mathrm{COMe}$ & -130.927 & -0.625 & 72.798 & 35.687 & 1.936 \\
\hline $3 \mathbf{i}$ & 4-pyridine & $\mathrm{COMe}$ & -125.392 & -1.157 & -3.427 & -125.293 & 2.382 \\
\hline $\mathbf{3 j}$ & 3-pyridine & $\mathrm{COMe}$ & -125.499 & -1.077 & -225.370 & 37.073 & 1.983 \\
\hline $3 \mathbf{k}$ & 3-1H-indole & $\mathrm{COMe}$ & -115.993 & -0.623 & -11.584 & 226.867 & 2.064 \\
\hline 31 & 2-methyl-5-furan & $\mathrm{COMe}$ & -168.641 & -0.827 & -68.720 & 168.542 & 1.964 \\
\hline $3 m$ & 5-furan-2-carbaldehyde & COMe & -191.133 & -1.244 & -344.990 & 244.203 & 1.999 \\
\hline $3 n$ & 5-furan-2-ylmethanol & $\mathrm{COMe}$ & -204.073 & -0.831 & 18.715 & 204.752 & 2.079 \\
\hline 30 & $\begin{array}{l}\text { 5-furan-2-ylmethyl } \\
\text { acetate }\end{array}$ & $\mathrm{COMe}$ & -246.568 & -0.966 & -396.949 & 114.470 & 1.064 \\
\hline $3 \mathbf{p}$ & $\begin{array}{l}\text { 5-furan-2-carboxylic } \\
\text { acid }\end{array}$ & $\mathrm{COMe}$ & -246.615 & -1.335 & -60.717 & -419.115 & 1.654 \\
\hline $3 \mathbf{q}$ & 2-nitro-5-furan & $\mathrm{COMe}$ & -164.122 & -1.800 & 320.183 & -818.085 & 2.998 \\
\hline $3 \mathbf{r}$ & 2-nitro-5-thiophene & $\mathrm{COMe}$ & -127.101 & -2.178 & 257.376 & $\begin{array}{c}- \\
1274.840\end{array}$ & 2.954 \\
\hline $3 s$ & 1,2-difluorobenzene & $\mathrm{COMe}$ & -219.127 & -1.257 & -53.420 & -232.088 & 1.914 \\
\hline $3 t$ & $\begin{array}{l}\text { (methylsulfonylmethyl) } \\
\mathrm{Ph}\end{array}$ & $\mathrm{COMe}$ & -246.637 & -0.973 & 9.587 & 295.576 & 2.236 \\
\hline $3 \mathbf{u}$ & pyrocatechol & $\mathrm{COMe}$ & -221.837 & -0.857 & 34.598 & 145.605 & 2.005 \\
\hline $3 \mathbf{v}$ & $p$-phenyl pivalate & $\mathrm{COMe}$ & -227.925 & -0.918 & -171.486 & 838.009 & 2.559 \\
\hline $4 \mathbf{a}$ & $\mathrm{Ph}$ & $\mathrm{CSMe}$ & -62.701 & -1.032 & 239.601 & 470.589 & 3.796 \\
\hline $4 b$ & 4-PhOH & $\mathrm{CSMe}$ & -108.102 & -1.030 & 434.518 & 323.365 & 3.854 \\
\hline
\end{tabular}




\begin{tabular}{|c|c|c|c|c|c|c|c|}
\hline 4d & 2-thiophene & $\mathrm{CSMe}$ & -52.838 & -1.185 & -266.866 & 105.475 & 2.430 \\
\hline $4 \mathrm{f}$ & 2-furan & $\mathrm{CSMe}$ & -88.649 & -1.063 & -235.789 & 126.774 & 2.203 \\
\hline $4 \mathbf{i}$ & 4-pyridine & $\mathrm{CSMe}$ & -55.413 & -1.133 & 23.571 & -39.428 & 2.796 \\
\hline $4 k$ & 3-1H-indole & CSMe & -45.871 & -1.041 & 279.246 & 270.114 & 3.690 \\
\hline $4 q$ & 5-nitro-2-furan & CSMe & -94.655 & -1.804 & 587.221 & -677.719 & 4.066 \\
\hline $5 \mathbf{a}$ & $\mathrm{Ph}$ & $\mathrm{CSNH}_{2}$ & -58.606 & -0.864 & 306.107 & 280.574 & 3.444 \\
\hline $5 b$ & 4-PhOH & $\mathrm{CSNH}_{2}$ & -104.003 & -0.868 & 511.264 & 111.319 & 3.503 \\
\hline $5 d$ & 2-thiophene & $\mathrm{CSNH}_{2}$ & -48.822 & -1.155 & -263.670 & 2.967 & 2.267 \\
\hline $5 f$ & 2-furan & $\mathrm{CSNH}_{2}$ & -84.638 & -0.909 & -228.015 & 21.757 & 1.856 \\
\hline $5 \mathbf{i}$ & 4-pyridine & $\mathrm{CSNH}_{2}$ & -50.552 & -1.111 & -183.405 & -343.462 & 1.898 \\
\hline $5 \mathbf{k}$ & 3-1H-indole & $\mathrm{CSNH}_{2}$ & -45.035 & -0.906 & -110.592 & 132.528 & 2.421 \\
\hline $5 v$ & $p$-phenyl pivalate & $\mathrm{CSNH}_{2}$ & -153.077 & -0.884 & 602.704 & 365.220 & 3.892 \\
\hline 6 & 2-furan & $\mathrm{COMe}$ & -122.747 & -0.510 & 0.971 & 193.289 & 1.847 \\
\hline 7 & 2-nitro-5-furan & $\mathrm{COMe}$ & -130.648 & -1.380 & -308.017 & -306.049 & 1.772 \\
\hline
\end{tabular}

${ }^{\mathrm{a}} \mathrm{C}$, concentration expressed in $\mathrm{mM} / \mathrm{L}$ of the drug molecules required for inhibition of $90 \%$ growth of $E$. fa ATCC 29212.

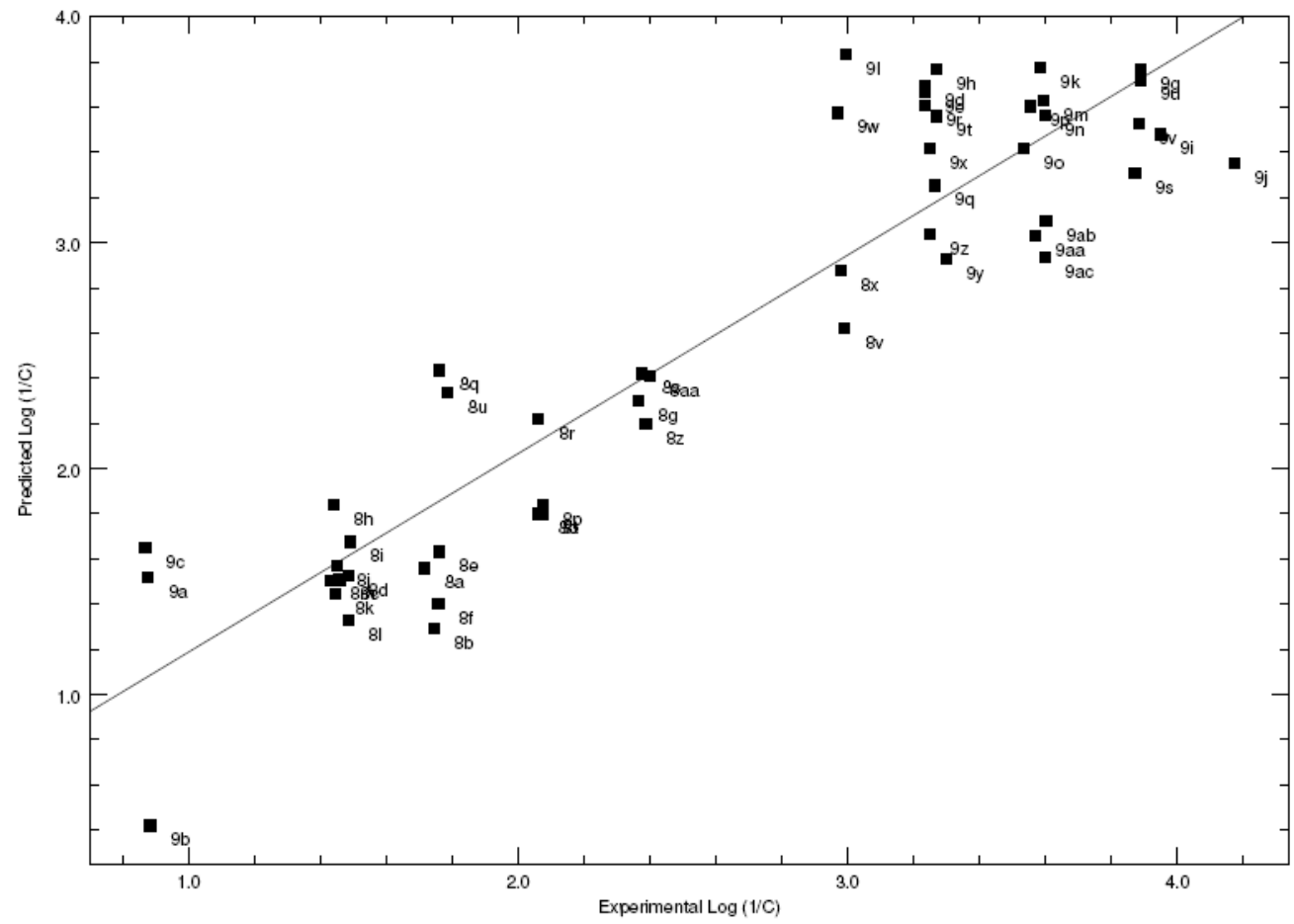

Figure 3. Prediction of antibacterial activity for the training and test set compounds $8-\mathbf{9}$ against E. fa ATCC 29212 using Equation 4. 


\section{Conclusions}

We can conclude that the 3D-QSAR equations based on HOF and LUMO energies enable us to predict the MIC value trends of oxazolidinone antibacterials and in many cases obtain close correlation with experimental MIC values. Calculation of the values of HOF and LUMO energies for compounds containing substitutions can also help to make predictions of antibacterial activities closer to experimental MIC values. For example, phenyloxazolidinones containing substitutions such as $\mathrm{CF}_{3}, \mathrm{COCF}_{3}, \mathrm{SO}_{2} \mathrm{CH}_{3}$, and $\mathrm{SOCHF}_{2}$ have a very low $\mathrm{HOF}$ (large negative) and high LUMO energies (high positive). Therefore, we can predict such compounds to show poor antibacterial activity based on predicted $\log (1 / \mathrm{C})$ values from our 3D-QSAR equations. Substitutions like $\mathrm{CN}$ or $\mathrm{NO}_{2}$ on furan or benzene rings lead to higher HOF (low negative value) and low LUMO energies (large negative) and are thus predicted to show good antibacterial activity for different sets of compounds and in different bacterial strains. These models provide us with a tool to make compounds with predictable antibacterial activities.

\section{Experimental Section}

General Procedures. TSAR 3D methods were used to derive 3D-QSAR equations as reported earlier. ${ }^{4}$ The structures were sketched using ChemDraw Ultra 5.0 (www.cambridgesoft.com) and were exported to TSAR 3.3 (www.accelrys.com). The three-dimensional structures of all the molecules were generated. Partial charges were derived using the Charge-2 CORINA 3D package in TSAR 3.3 and the geometries of the molecules were optimised using the Cosmic module of TSAR. The calculations were terminated if the energy difference or the energy gradient were smaller than $1 \mathrm{e}-005$ and $1 \mathrm{e}-010 \mathrm{kcal} / \mathrm{mol}$ respectively. The respective MIC values in $\mathrm{mg} / \mathrm{mL}$ of compounds 8-9 were converted to $\log (1 / \mathrm{C})$, where $\mathrm{C}$ is the concentration expressed in $\mathrm{mM} / \mathrm{L}$ of the drug molecule required for $90 \%$ inhibition of the growth of the microorganism. Data for inactive compounds in the ranges $>16 \mu \mathrm{g} / \mathrm{mL},>32 \mu \mathrm{g} / \mathrm{mL}$ and $>64$ $\mu \mathrm{g} / \mathrm{mL}$ are treated as $=16 \mu \mathrm{g} / \mathrm{mL}, 32 \mu \mathrm{g} / \mathrm{mL}$ and $64 \mu \mathrm{g} / \mathrm{mL}$, respectively, for the training data set and $\mathrm{r}^{\wedge} 2$ determination.

Molecular descriptors for the entire molecules were calculated with TSAR 3.3. Vamp, a semiempirical molecular orbital package in TSAR 3.3, was used to calculate electronic properties, including heats of formation, HOMO and LUMO energies, polarizability and multipole components, and to perform structure optimizations in vacuo using default parameters and using the Hamiltonian method PM3. Descriptors with the same values for all compounds 89 were discarded. A pair-wise correlation analysis of the remaining descriptors was performed. Regression models were built using descriptor subsets containing only one of these highly correlated descriptors. To develop QSAR models, stepwise MLR analysis with leave-one-out (LOO) cross-validation was applied to the data set. The approach used for the prediction of 
antibacterial activities is a simple regression analysis. We refer to these procedures as $3 \mathrm{D}$ QSAR methods.

\section{Acknowledgements}

I am grateful to Dr. Brijesh Srivastava, Sr. Scientist and Dr. Vidya Lohray, former VicePresident, Zydus Research Centre for their encouragement and continuous support. I also acknowledge Dr. Ricardo Mancera, School of Biomedical Sciences, Curtin University of Technology for his help with the manuscript.

\section{References}

1. Service, R. F. Science 1995, 5237, 724.

2. (a) Swaney, S. M.; Aoki, H.; Ganoza, M. C.; Shinabarger, D. L. Antimicrob. Agents Chemother. 1998, 12, 3251. (b) Brickner, S. J. Current Pharmaceutical Design 1996, 2, 175. (c) Zurenko, G. E.; Yagi, B. H.; Schaadt, R. D.; Allison, J. W.; Kilburn, J. O.; Glickman, S. E.; Hutchinson, D. K.; Barbachyn, M. R.; Brickner, S. J. Antimicrob. Agents Chemother. 1996, 4, 839.

3. (a) Gopalakrishnan, B.; Khandelwal, A.; Rajjak, S. A.; Selvakumar, N.; Das, J.; Trehan, S.; Iqbal, J.; Kumar, M. S. Bioorg. Med. Chem. 2003, 12, 2569. (b) Karki, R. G.; Kulkarni, V. M. Bioorg. Med. Chem. 2001, 12, 3153. (c) Katritzky, A. R.; Fara, D. C.; Karelson, M. Bioorg. Med. Chem. 2004, 11, 3027. (d) Pae, A. N.; Kim, S. Y.; Kim, H. Y.; Joo, H. J.; Cho, Y. S.; Choi, K. I.; Choi, J. H.; Koh, H. Y. Bioorg. Med. Chem. Lett. 1999, 18, 2685. (e) Tokuyama, R.; Takahashi, Y.; Tomita, Y.; Tsubouchi, M.; Yoshida, T.; Iwasaki, N.; Kado, N.; Okezaki, E.; Nagata, O. Chem. Pharm. Bull. (Tokyo) 2001, 4, 353.

4. Lohray, B. B.; Gandhi, N.; Srivastava, B. K.; Lohray, V. B. Bioorg. Med. Chem. Lett. 2006, 14, 3817.

5. Das, B.; Rudra, S.; Yadav, A.; Ray, A.; Rao, A. V.; Srinivas, A. S.; Soni, A.; Saini, S.; Shukla, S.; Pandya, M.; Bhateja, P.; Malhotra, S.; Mathur, T.; Arora, S. K.; Rattan, A.; Mehta, A. Bioorg. Med. Chem. Lett. 2005, 19, 4261.

6. Jang, S. Y.; Ha, Y. H.; Ko, S. W.; Lee, W.; Lee, J.; Kim, S.; Kim, Y. W.; Lee, W. K.; Ha, H. J. Bioorg. Med. Chem. Lett. 2004, 15, 3881. 